\title{
Ileitis after colectomy for ulcerative colitis or carcinoma
}

\author{
A Hallak, M Baratz, M Santo, Z Halpern, M Rabau, N Werbin, T Gilat
}

\begin{abstract}
An ileitis developing years or months after ileostomy was recognised and described many years ago but has rarely been mentioned since. We describe nine patients with a non-specific preanastomotic ileitis that developed years after colectomy in patients operated on for ulcerative colitis or carcinoma. The disease developed after various types of reconstruction: ileorectal, ileoanal, with or without pouch. The diseased ileum showed inflammation, erosions, ulcerations, and sometimes strictures. The disease course ranged from asymptomatic to severe pain and diarrhoea. No specific cause could be shown. Granulomas, infectious agents or ischaemic changes were not found. The anastomosis was always patent. Three patients have had numerous episodes of intestinal obstruction presumably because of adhesions. Seven of nine patients were female. The response to steroids, 5-aminosalicyclic acid preparations, and methotrexate was poor, three patients responded well to azathioprine. The condition is not rare and the cause remains unknown. (Gut 1994; 35: 373-376)
\end{abstract}

Various diseases of the colon are treated, when necessary, by removal of the organ. The resection may be complete (proctocolectomy) or incomplete. The number of potential reconstructions has increased in recent years and includes ileostomy with or without pouch,,$^{1-4}$ and ileorectal ${ }^{5}$ or ileoanal anastomosis with or without pouch. ${ }^{6-9}$ The common denominator of all these procedures is that the terminal ileum is reanastomosed in a non-physiologic environment. Many decades ago it was recognised that the ileum in this situation may develop disease. ${ }^{10-12}$ Thayer and Spiro in $1962^{13}$ described this disease in the specific situation of ileostomy and coined the term 'prestomal ileitis'. This entity has very rarely been mentioned in past decades as exemplified in a recent review. ${ }^{14} \mathrm{We}$ have seen in the last three years nine cases of unexplained ileitis in association with colectomy for various causes and reconstructions of various types and now report our findings.

Patients

CASE NO 1

A 49 year old woman had a subtotal colectomy for ulcerative colitis 12 years ago. An ileorectal anastomosis with diverting ileostomy was performed. One month after closure of the ileostomy, intestinal obstruction as a result of adhesions necessitated a third operation with lysis of adhesions. The postoperative course was uneventful, and the remaining proctitis responded well to local and oral treatment. A flexible sigmoidoscopy performed three years after the colectomy disclosed a very mildly active proctitis affecting the entire rectum up to the anastomotic area at $18 \mathrm{~cm}$. The anastomosis was widely open and the ileal mucosa had a normal appearance. A barium transit examination showed some dilatation of the small bowel close to the anastomosis with unexplained delay in the transit. In the next six years the patient experienced three additional episodes of intestinal obstruction, all of them treated conservatively. A routine sigmoidoscopy performed nine years after the colectomy showed for the first time the existence of patchy ileitis with a thickened mucosa in which superficial erosions and pseudopolyps were seen extending from the anastomotic line to $40 \mathrm{~cm}$ into the small intestine. The microscopic examination showed shortening, widening, and in some areas flattening of the villi (Fig 1, right side). The surface epithelium was permeated by polymorphs and in some areas erosion were present. The stroma contained numerous chronic and acute inflammatory cells, lymphocytes, plasma cells, and polymorphonuclear neutrophils. Cryptitis and crypt abscesses were seen (Fig 1 left side and arrow). The microscopic findings were consistent with non-specific ileitis. The rectal stump was normal except for a few erosions in the proximal rectum near the anastomosis. These were noted on several examinations. The patient had been receiving 5-aminosalicylic acid (5-ASA) (mesalazine) treatment throughout. Granulomas were absent. Specific stains and electron microscopy in the search for fungi, cytomegalic inclusions, herpes virus and other viruses were negative. Serological tests were positive for cytomegalovirus and herpes virus but remained at low titres. Examination of the colectomy specimen reconfirmed the diagnosis of ulcerative colitis. The patient at that point had severe ileitis of uncertain cause and started having left abdominal pains in addition to her frequent loose bowel movements.

A course of metronidazole was added to the continuous treatment with 5-ASA without any benefit and was stopped. She was then given steroids orally. The ileitis did not respond. Azathioprine was tried for a short time with symptomatic and endoscopic response but had to be stopped because of peripheral neuropathy, which resolved after the drug was withdrawn. The patient has been receiving methotrexate for the last 10 months without apparent benefit. She now has abdominal pains attributable to the ileitis and two incomplete strictures were recently 


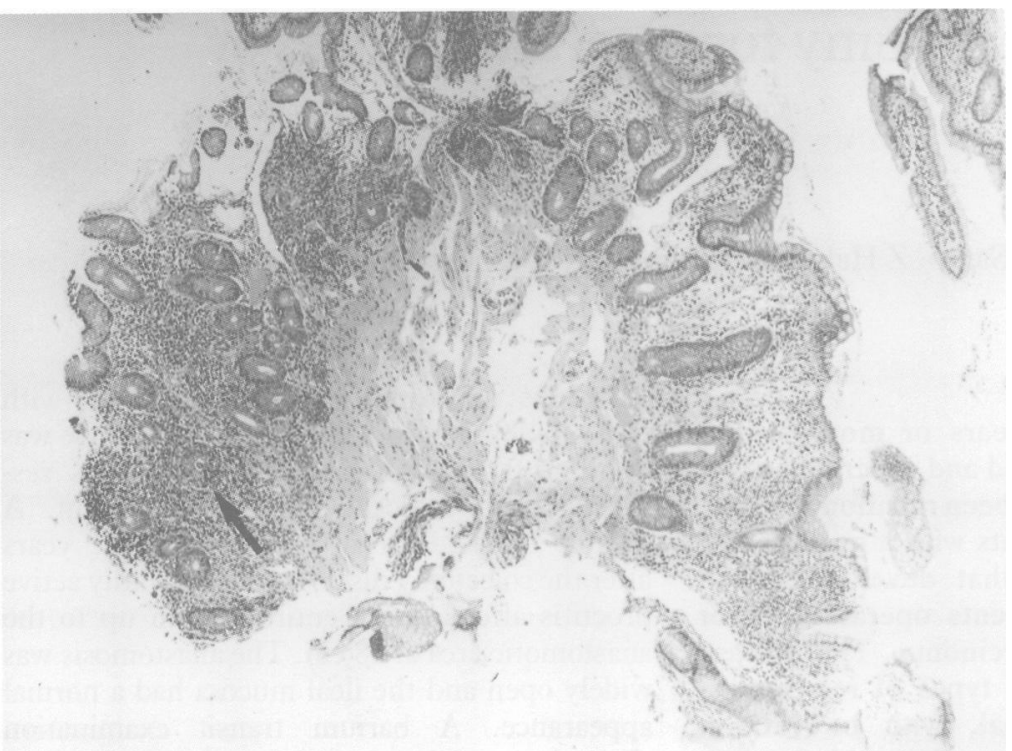

Figure 1: Biopsy specimen of inflamed ileum case 1. Right side: flattening of the villi; left side: superficial erosions, appreciable inflammation in the stroma, cryptitis, and crypt abscess (arrow). (Haematoxylin and eosin, original magnification $\times 100)$

found in the diseased ileum. The proximal extent of inflammation cannot be ascertained because of the strictures, which do not permit passage of the endoscope. She is currently receiving 6 mercaptopurine $50 \mathrm{mg}$ daily with symptomatic improvement and as yet no neuropathy.

CASE NO 2

A 62 year old female patient had subtotal colectomy with ileorectal anastomosis for a right sided colonic carcinoma and large sigmoid

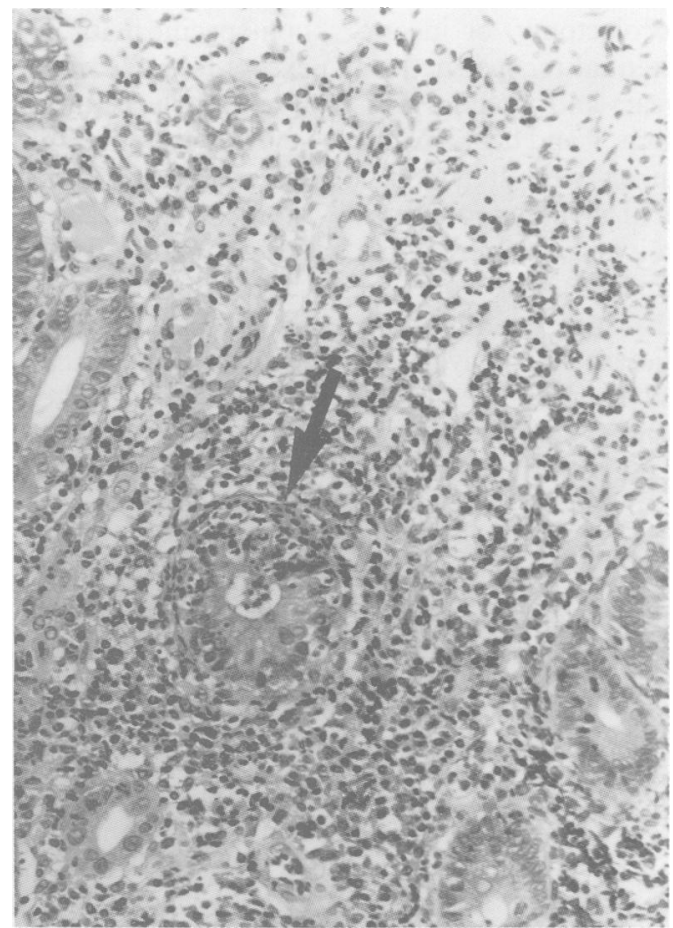

Figure 2: Higher magnification of Fig 1 (the left side). Inflammatory infiltrate in the stroma, cryptitis, and crypt abscess (arrow). (Haematoxylin and eosin, original magnification $\times 350$ adenoma 13 years ago. Her past history included a salpingo-oophorectomy at age 35 , and cholecystectomy at age 48 . In the first five years after surgery, the patient suffered from recurrent episodes of subacute intestinal obstruction because of adhesions. An extensive series of investigations was negative except for an appreciable delay in small bowel emptying when tested by barium transit. Adhesions were considered to be the cause of her symptoms and she was treated conservatively. She was asymptomatic for about four years, when three other episodes of subileus occurred within the last six months. Another investigation was normal, and the barium transit was considered normal this time. Repeated sigmoidoscopic examinations showed the anastomotic area to be widely open. The preanastomotic ileum, however, was seen to be mildly inflamed and reddened on the last three examinations. There were some superficial erosions and ulcerations. Biopsy specimens taken from that area showed shortening and widening of the villi, and the stroma contained an increased number of acute and chronic inflammatory cells. The patient is completely asymptomatic in relation to this macroscopic and microscopic ileitis.

\section{CASE NO 3}

A 35 year old female patient had a total colectomy with ileoanal anastomosis (without pouch) for ulcerative colitis 20 years ago. The patient did fairly well until six years ago, when she started experiencing severe episodes of diarrhoea. Sigmoidoscopic examination showed severe ileitis affecting the distal preanastomotic $20 \mathrm{~cm}$. The mucosa was inflamed with redness, erosions, and pseudopolyps; the lumen was constricted 17 $\mathrm{cm}$ proximally to the anastomosis. The inflammation was found on both sides of the stricture. The microscopic findings were similar to case 1 . In addition to cryptitis, crypt abscesses were seen in this patient. Careful search did not show the presence of granulomas and a diagnosis of Crohn's disease was not confirmed. There was no evidence of ischaemia. Parasites, bacteria, and viruses were not found either by histological examination and electron microscopy or by bacteriological stool examinations. Review of the proctocolectomy specimen (at the Mayo Clinic) reconfirmed the diagnosis of ulcerative colitis. The patient was given 5-aminosalicylic acid enterally and locally (with enemas) and then was given steroids without success. She improved dramatically, however, clinically and endoscopically when azathioprine treatment was started. Even the stricture resolved. The patient planned pregnancy and azathioprine was discontinued. A severe clinical and endoscopic relapse occurred. Azathioprine treatment was started again with resultant remission of the disease. She has now been on this treatment for over 18 months and is still in remission.

\section{CASE 4}

A 78 year old male patient had subtotal colectomy and high ileo-anastomosis for carcinoma in the ascending colon and severe diverticular disease 


\begin{tabular}{|c|c|c|c|c|c|c|c|c|c|c|c|}
\hline No & Age & $M / F$ & Diagnosis & Surgery & $\begin{array}{l}\text { Intervals } \\
\text { (years) }\end{array}$ & $\begin{array}{l}\text { Ileitis† (years) } \\
\text { Asymptomatic }\end{array}$ & Symptomatic & Endoscopy & Effective & Treatment & Ineffective \\
\hline 1 & 49 & $\mathrm{~F}$ & UC & IRA & 9 & 2 & 1 & Ileitis proctitis in remission & AZA & \multirow{5}{*}{ none } & 5ASA Steroids MND MTX \\
\hline 2 & 62 & $\mathbf{F}$ & $\mathrm{Ca}$ & IRA & 10 & 3 & 0 & Ileitis $15 \mathrm{~cm}$ & & & \\
\hline 3 & 35 & $\mathrm{~F}$ & UC & IAA & & 0 & & Severe ileitis $20 \mathrm{~cm}$ & AZA & & 5ASA \\
\hline 4 & 78 & $M$ & CA & IRA & $1 \mathrm{mo}$ & 0 & $4 \mathrm{mo}$. & Ileitis $15 \mathrm{~cm}$ & & & MND 5ASA \\
\hline 5 & 40 & $\mathrm{~F}$ & UC & $\begin{array}{l}\text { IAA } \\
\text { S-pouch }\end{array}$ & 5 & 0 & & Severe ileitis Mild pouchitis & & & MND \\
\hline 6 & 49 & $M$ & UC & IRA & 10 & 2 & 0 & Ileitis $30 \mathrm{~cm}$ & \multirow{4}{*}{$6 \mathrm{MP}$} & \multirow{3}{*}{ none } & 5ASA \\
\hline 7 & 44 & $\mathbf{F}$ & UC & IRA & 14 & 1 & 0 & Ileitis $30 \mathrm{~cm}$ & & & \multirow{3}{*}{ 5ASA MND Steroids } \\
\hline 8 & 48 & $F$ & UC & IAA & 1 & 0 & 1 & $\begin{array}{l}\text { Severe ileitis } 30 \mathrm{~cm} \\
\text { Mild pouchitis }\end{array}$ & & & \\
\hline 9 & 32 & $\mathrm{~F}$ & UC & IRA & 12 & $1 / 2$ & 0 & Ileitis $40 \mathrm{~cm}$ & & none & \\
\hline
\end{tabular}

$\star$ Interval between colectomy and ileitis; + time since diagnosis of ileitis; $\mathrm{UC}=$ ulcerative colitis, $\mathrm{Ca}=\mathrm{carcinoma}$, IRA $=\mathrm{ileorectal}$ anastomosis, IAA $=\mathrm{ileoanal}$ anastomosis, $\mathrm{AZA}=$ azathioprine, $\mathrm{MTX}=$ methotrexate, $\mathrm{MND}=$ metronidazole, $5 \mathrm{ASA}=5$ aminosalicyclic acid, $6 \mathrm{MP}=6$ mercaptopurine.

in the sigmoid. The postoperative course was uneventful. One month after operation, however, he started experiencing severe diarrhoea, with up to 20 bowel movements/day. A barium transit did not disclose any pathological pattern. The anastomosis was widely open and located at $20 \mathrm{~cm}$ from the anal verge. At sigmoidoscopy examination the rectal mucosa was normal. Proximal to the anastomosis for about $15 \mathrm{~cm}$ the distal ileal mucosa was considerably inflamed and reddened with multiple aphtoid ulcerations. There was no stricture. Biopsy specimens taken from this area showed chronic inflammatory infiltrate with flattening of the villi and ulcerations. Granulomas were not found. Bacteriological and parasitological examinations of stools were repeatedly negative. The patient was treated with metronidazole and 5-ASA with clinical, but not endoscopic improvement. The patient has now been followed up for about four months for ileitis without endoscopic or histological improvement.

The Table provides details of all nine patients with postcolectomy ileitis currently under our care. They were all diagnosed in the last three years.

\section{Discussion}

When we first encountered case 1 with ileitis after ileorectostomy for ulcerative colitis, we were baffled. Examination of the colectomy specimen reconfirmed the diagnosis of ulcerative colitis. The rectal stump was in remission, there was no obstruction at the anastomosis, and specific causes such as herpes or viral inclusions, parasitic or ischaemic disease could not be confirmed. Within two years, however, we encountered similar cases without inflammatory bowel disease (colectomy and ileorectal anastomosis for tumours); direct ileoanal anastomosis without rectal stump or pouch; ileoanal anastomosis with pouch. Therefore, the inflammation was not connected to pre-existing colitis or to the type of reconstruction, or to the presence of a rectal stump or pouch. The pathological spectrum of disease was quite wide, ranging from mild asymptomatic redness of the mucosa with only histological ileitis to an obviously inflamed mucosa with or without ulcers. Strictures also developed in the ileum with disease present both proximally and distally to the incomplete strictures. The disease was predominantly diffuse though occasionally patchy and could change from one form to the other in the same patient over a period of months. As we could not find a specific cause or any specific operation or pre-existing disease associated with this ileitis, we eventually recognised that it was associated with colectomy and reanastomosis only. Review of published work showed that this disease, albeit in a limited number of surgical conditions, had been recognised many years earlier. Publications in the 1950 s reported ileitis in $4 \cdot 1$ to $5 \cdot 5$ per cent of patients with ileostomy. ${ }^{10-18}$ Thayer and Spiro ${ }^{13}$ defined 'prestomal ileitis' as an inflammatory process that affects the ileum proximal to the ileostomy. They described two distinct types of prestomal ileitis: acute, starting soon after colectomy, and latent, beginning months or years later. Knill-Jones et al reported in 1970 five cases of prestomal ileitis after colectomy for ulcerative colitis. ${ }^{19}$ As the condition is not confined to ileostomy and may occur after various types of reconstruction, we suggest postcolectomy ileitis as a more appropriate definition.

The cause of this ileitis is presently unproved and permits only speculation. It does not seem to be related to an infectious agent because multiple cultures and electron microscopic studies consistently failed to show any bacteria, viruses or parasites. Granulomas as well as inclusion bodies were also not found.

Is this proximal spread of ulcerative colitis to the ileum similar to backwash ileitis? Unlike backwash ileitis, which always develops while the diseased colon is in place, the ileitis described here developed months to many years after removal of the diseased colon. It also developed in two patients who had not had ulcerative colitis. We therefore do not think this possibility to be likely.

An ischaemic cause is always tempting and difficult to refute. Biopsy specimens from the damaged ileum, however, did not show any ischaemic or thrombotic pattern. In the past reports, the resected ileum did not show any ischaemia. In our series, patient no 7 had selective angiography of the superior mesenteric artery and the vascular pattern was normal. Ileitis occurred in different conditions related to the disease causing colectomy (for example ulcerative colitis, carcinoma) and to the type of surgery. A mechanical cause cannot be discarded. Indeed, several of our patients did suffer from recurrent episodes of partial or complete mechanical obstruction (presumably because of 
adhesions). Nevertheless, most of the patients did not experience any obstruction and in all patients the anastomotic area was found to be widely open by radiography and endoscopy. It is remarkable, though unexplained, that seven of our nine cases were female.

A variety of reports ${ }^{2021}$ have described inflammatory lesions of the small bowel in patients treated with non-steroidal anti-inflammatory drugs. In our group of patients none experienced arthritic pains or was receiving non-steroid antiinflammatory drug treatment.

What about treatment? In the first case, 5ASA as well as short term steroid treatment were ineffective. Azathioprine seemed to induce symptomatic as well as endoscopic improvement. Methotrexate was unhelpful. Case no 3 also did not respond to 5-ASA and steroids, and responded dramatically to azathiorine. She relapsed when azathioprine was stopped, and responded to its reinstitution. In case no $76 \mathrm{MP}$ seemed to be beneficial but had to be withdrawn because of side effects. Three cases are not sufficient for drawing conclusions, but azathioprine should be subjected to a controlled trial in this condition.

In conclusion, postcolectomy ileitis seems to be a well defined entity as described many years ago and in this series. The clinical presentation is variable, from the absence of complaints to severe diarrhoea, abdominal pains, and dehydration. The endoscopic features include inflammation, ulcerations, thickening of the wall, pseudopolyps, and strictures affecting the ileum from the anastomotic area to a variable distance proximally in a diffuse or patchy manner. The cause is unknown at the present and the response to treatment is poor. The condition does not seem to be rare.
1 Ripstein CB, Miller GG, Gardner CM. Results of the surgical treatment of ulcerative colitis. Ann Surg 1952; 135: 14-8.

2 Counsell PB, Goligher JC. The surgical treatment of ulcerative colitis. Lancet 1952; ii: 1045-50.

3 Brooke BN. The management of an ileostomy. Lancet 1952; ii: $102-4$.

4 Kock NG, Intra abdominal 'reservoir' in patients with permanent ileostomy; preliminary observations on a procedure resulting in fecal 'continence' in five ileostomy patients. Arch Surg 1986; 99: 223-30.

5 Aylett SO. Three hundred cases of diffuse ulcerative colitis treated by total colectomy and ileo-rectal anastomosis. $B M \mathcal{F}$ 1966; 1: 1001-5.

6 Fonkalsrud EW. Total colectomy and endorectal ileal pullthrough with internal ileal reservoir for ulcerative colitis. Surg Gynecol Obstet 1980; 150: 1-8

7 Utsunomiya J, Iwama T, Imajo $M$, Matsuo S, Sawai S, Yaegashi K, et al. Total colectomy, mucosal proctectomy and ileoanal anastomosis. Dis Colon Rectum 1980; 23: 459-65.

8 Nicholls RJ, Lubowski DZ. Restorative proctocolectomy: The four loop (W) reservoir. $B M \mathcal{F}$ 1987; 74: 564-6.

9 Parks AG, Nicholls RJ, Bellivea P. Proctocolectomy with ileoanal reservoir and anal anastomosis. Br $\mathcal{F}$ Surg 1980; 67 : 533-8.

10 Warren R, McKittrick LS. Ileostomy for ulcerative colitis: technique, complications and management. Surg Gynecol Obstet 1951; 93: 555-67.

11 Rogers AG, Bargen JA, Black BM. Chronic ulcerative colitis: early and late experiences of 124 patients with ileal stomas. Garly and late experiences of 124 patogy 1954; 27 : 383-98.

12 Lyons AS, Garlock HJ. The complications of ileostomy. Surgery 1954; 36: 784-9.

13 Thayer WR, Spiro HM. Ileitis after ileostomy: prestoma ileitis. Gastroenterology 1962; 42: 547-54.

14 Scott AD, Phillips RKS. Ileitis and pouchitis after colectomy for ulcerative colitis. Br $\mathcal{Y}$ Surg 1989; 76: 688-9.

15 Colcock BP, Mathiesen WL. Complications of surgical treatment of chronic ulcerative colitis. Arch Surg 1956; 72: 399 404.

16 Tolstedt GE, Bell JW. Intestinal obstruction following total colectomy for ulcerative colitis. Ann Surg 1961; 153: 241-5.

17 Brown CH, Turnbull RB, Diaz R. Ileorectal anastomosis in ulcerative colitis - results in 27 patients. Am f Dig Dis 1962; ulcerative

18 Turnbull RB, Weakley FL, Farmer RG. Ileitis after colectomy and ileostomy for nonspecific ulcerative colitis: report of 35 cases. Dis Colon Rectum 1964; 7: 427-35.

19 Knill-Jones RP, Morson B, Williams R. Prestomal ileitis: clinical and pathological findings in five cases. Q J Med 1970; 154: 287-97.

20 Lang J, Price AB, Levi AJ, Burke H, Gumpel IJM, Bjarnason I. Diaphragm disease: pathology of disease of the small intestine induced by non-steroidal anti-inflammatory drugs. f Clin Pathol 1988; 41: 516-26.

21 Allison MC, Howatson AG, Torrance CJ, Lee FD, Russell RI. Gastrointestinal damage associated with the use of nonsteroidal anti-inflammatory drugs. $N$ Engl F Med 1992; 327: 749-54. 